\title{
Animal models of memory impairment
}

\author{
MICHELA GALLAGHER \\ Department of Psychology, University of North Carolina at Chapel Hill, Chapel Hill, NC 27599, USA \\ (mgallagh@email.unc.edu)
}

\begin{abstract}
SUMMARY
Memory impairment in the elderly resembles a mild temporal lobe dysfunction. Alterations in the hippocampal formation are also a probable basis for cognitive deficits in some animal models of ageing. For example, aged rats are impaired in hippocampal-dependent tests of spatial memory. Recent studies have revealed considerable structural integrity in the aged hippocampus, even in aged rats with the most impaired spatial memory. In contrast, atrophy/loss of cholinergic neurons in the basal forebrain and deficiency in cholinergic transduction in hippocampus correlate with the severity of spatial memory impairment in aged rats. This evidence supports the longstanding view that age-related loss of memory has a cholinergic basis. In this context, it is somewhat surprising that the use of a selective cholinergic immunotoxin in young rats to further test this hypothesis has revealed normal spatial memory after removing septo-hippocampal cholinergic neurons. Young rats with immunotoxic lesions, however, have other behavioural impairments in tests of attentional processing. These lines of research have implications for understanding the neurobiological basis of memory deficits in ageing and for selecting an optimal behavioural setting in which to examine therapies aimed at restoring neurobiological function.
\end{abstract}

\section{INTRODUGTION}

Memory impairment can occur as a relatively isolated sequel to brain damage. As reviewed elsewhere in this volume, studies of human amnesia and investigations of animal models for those conditions have disclosed a wealth of information about the organization of memory in the brain (see Squire \& Zola, this volume). Neurobiological systems in mammals are specialized for distinct forms of memory. In particular, components of the brain's medial temporal lobe support the formation of memories that are explicitly accessible as remembered facts and events, often referred to as declarative memory.

The study of memory, based on relatively circumscribed cases of human amnesia and related animal models, has also informed our understanding of the relevant pathology underlying the failure of memory in other conditions. A recognition that neurodegeneration occurs at several critical sites in the medial temporal lobe, including the hippocampus, in Alzheimer's disease provides a basis for loss of cognitive functions that depend on those structures (Hyman et al. 1984, 1987; West et al. 1994). Because Alzheimer's disease and other forms of dementia in the elderly occur against a background of brain ageing, recent research using animal models has also helped to distinguish between such pathological conditions and normal ageing. Recent findings, which will be highlighted in this short account of that research, provide new evidence for greater neurobiological preservation in the aged brain than was previously appreciated. Animal models also promise to advance our under- standing of the functional implications of those changes that occur in the brain during normal ageing.

\section{ANIMAL MODEL OF HIPPOGAMPAL - DEPENDENT GOGNITIVE IMPAIRMENT}

Considerable advances have occurred in the development of animal models geared to examining the condition of specific neurobiological systems during ageing. An important feature of such models is that functional assessments are made on behavioural tasks that require the integrity of the neurobiological system of interest. One widely used model capitalizes on the observation that tasks measuring spatial learning and memory are particularly sensitive to both hippocampal damage (Morris et al. 1982) and ageing in the rat (for reviews, see Gage et al. 1984; Gallagher \& Rapp 1997). In a frequently used water maze apparatus, young and aged rats are tested in two standardized training protocols: one that relies on the functional integrity of the hippocampal system, and another that involves many of the same sensorimotor demands but does not require the medial temporal lobe system.

The water maze apparatus consists of a large, circular pool filled with water that has been made opaque through the addition of powdered milk or some other substance. In the hippocampal-dependent 'hidden platform' version of the task, rats are trained to find a camouflaged escape platform that is positioned just below the water surface. The location of this platform remains constant from trial to trial. Because there are no local cues that mark the position 


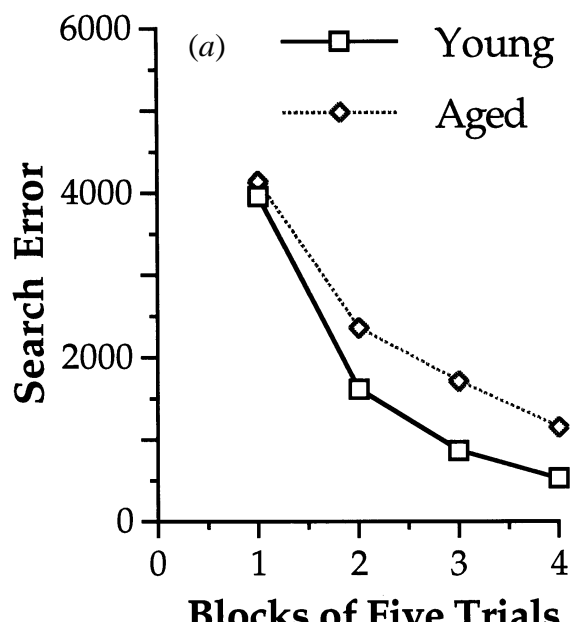

Blocks of Five Trials

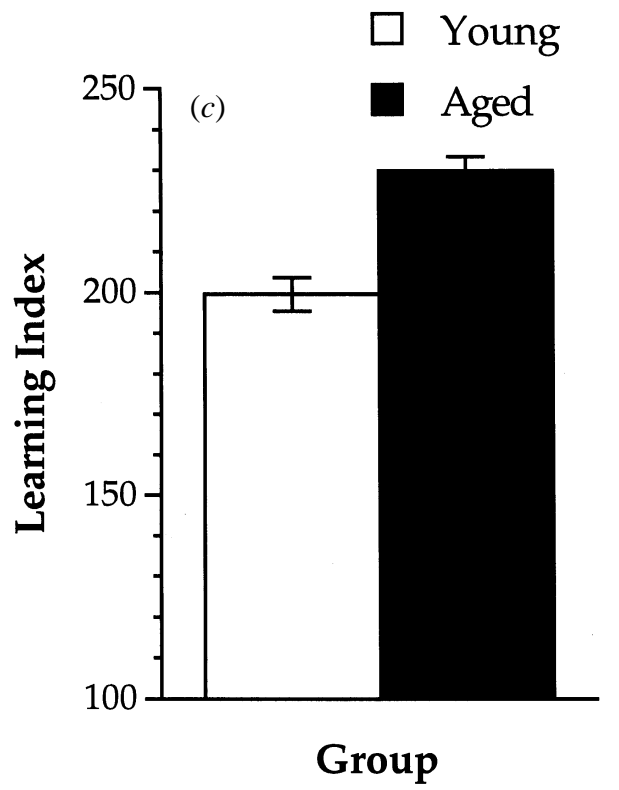

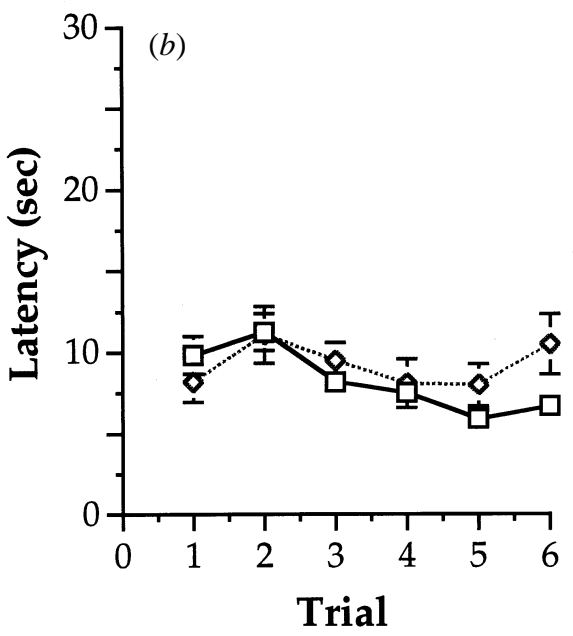

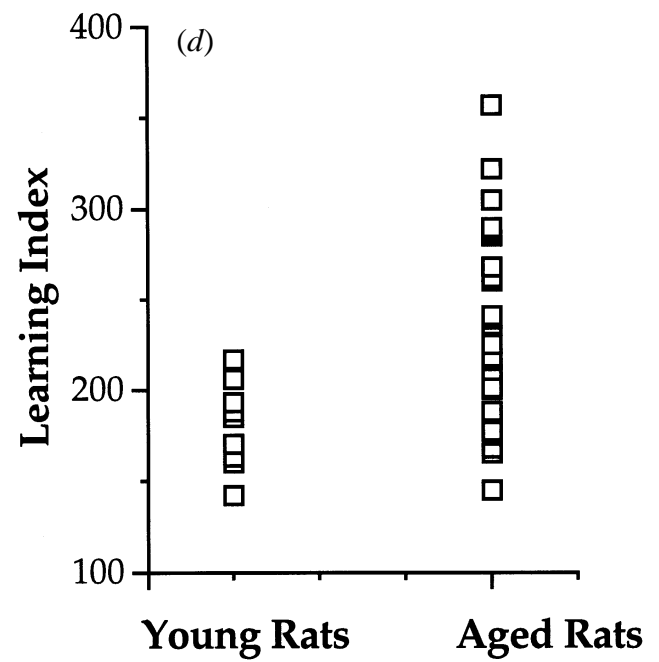

Figure 1. Water maze performance in young (6 months) and aged (28-29 months) male Long-Evans rats. (a) Age-dependent impairment during training trials in the hidden platform task that depends on spatial learning. The dependent measure, search error, is based on a calculation of average distance from the platform every second during the training trials. In contrast, aged and young rats perform with equal proficiency during cue training using a visible escape platform (shown in $(b)$ ). These graphs present archival data for 96 young and 128 aged male Long-Evans rats trained in a standard protocol described in Gallagher $e t$ al. (1993). Data from interpolated probe trials also show an age-dependent deficit in acquisition of a spatially guided search strategy in the hidden platform task (shown in $(c)$ ), where lower values reflect a more accurate search in the escape platform location. Individual scores derived from these probe trials are shown in the lower graph on the right $(d)$ for a sample of young and aged rats. Note the individual differences among the aged rats, with some subjects performing as well as young and others falling outside the entire range of young performance. (With permission from Gallagher et al. 1993.)

of the platform, the rat's ability to locate it efficiently depends on using a spatial configuration of extramaze cues surrounding the pool. Indeed, young rats can learn to swim directly to the escape platform within relatively few training trials from any of a number of start locations at the perimeter of the pool. Probe trials are interpolated during the course of training to assess the development of a spatially guided learning strategy. At the completion of this testing rats can be assessed for their ability to locate a visible platform, unaided by spatial location within the maze environment. This cue-training version of the task is unaffected by hippocampal damage (Morris et al. 1982). The consistent finding across our studies, as well as experiments conducted in other laboratories, is that a substantial proportion of aged rats exhibit behavioural deficits qualitatively similar to the effects of hippocampal damage. Other rats of the same chronological age perform as well as young rats on the hippocampal-dependent version of the water maze task. The data in figure 1 show an age-dependent impairment in spatial learning (but not cue learning) and also illustrate the phenomenon of individual differences among aged rats (Gallagher et al. 1993).

Behavioural assessment provides a useful context for defining neurobiological alterations in the aged brain that contribute to cognitive decline (for reveiws, see Gallagher \& Rapp 1997; Rapp \& Amaral 1992). By 
this approach, relevant neural systems in brains from aged rats with identified impairment can be compared with the condition of those systems in rats of the same age that exhibit preserved function. Such comparisons are likely to be more sensitive than merely comparing aged rats with young subjects, given that only a proportion of aged animals are impaired. In addition, it is possible that certain biological changes that occur during ageing have relatively little consequence for the maintainance of functional capacity. Thus, identification of neurobiological alterations that are closely coupled to cognitive decline may point to those effects of ageing that are likely to compromise adaptive capacities in the elderly.

\section{PRESERVATION OF PRINGIPAL NEURONS IN HIPPOGAMPUS OF AGED RATS}

The basis for age-related decline in hippocampaldependent functions, as illustrated by spatial learning impairment, has been attributed to neurodegeneration within this system (Landfield et al. 1981; Meaney et al. 1988). Loss of neurons in the hippocampus of aged rats was originally inferred from studies that sampled neuron density in the principal cell fields of this structure. Previous studies linking neuron loss to functional decline in ageing have reported a similar result in rats characterized for spatial learning ability. For example, CA3/2 neuron density was reported to decline by as much as $30 \%$ in behaviourally impaired aged rats (Issa et al. 1990). The water maze task used to characterize behavioural impairment in that study was the same used to test aged rats, as shown in figure 1. Subsequent research, however, has provided compelling evidence that frank neuron degeneration is not an inevitable feature of hippocampal ageing, even in rats that exhibit substantial impairment in spatial learning ability (Rapp \& Gallagher 1996; Rasmussen et al. 1996). In this recent work, a stereological examination yielded counts for total number of principal neurons in the hippocampus using the optical fractionator technique. For the granule cell layer, and in the CA3/2 and CAl pyramidal cell fields, total neuron number was statistically indistinguishable in young subjects, aged rats with behavioural deficits predictive of hippocampal dysfunction and aged rats that performed as accurately as younger individuals (Rapp \& Gallagher 1996). These findings indicate that previous reports of age-related changes in cell density are not likely to result from neuron loss. Although some undefined difference may exist in the aged rats used in these studies, clearly spatial learning impairment can occur in the absence of neuron loss.

The stereological results obtained in the rodent model of ageing are consistent with preliminary evidence in monkeys and humans, indicating that the principal neurons of the hippocampus are preserved in normal ageing (West 1993; West et al. 1993). Other recent investigations of the rodent model in our study population have indicated that a number of proteins used for neurocommunication in this system are also present in similar quantity in aged rats, irrespective of their spatial learning ability. We have reported that the amount of hippocampal $\mathrm{ml}$ and $\mathrm{m} 3$ receptor proteins, which serve as postsynaptic cholinergic receptors of the muscarinic type, are unaffected by age and uncorrelated with behavioural decline in ageing (Chouinard et al. 1995). Similarly, the amount of NR1, NR2a and $\mathrm{NR} 2 \mathrm{~b}$ protein in the hippocampus of behaviourally characterized animals is unaffected by age or behavioural status (B. B. Wolfe, personal communication). These proteins comprise subunits for a type of ionotropic glutamate receptor, known as the N-methyl-Daspartate (NMDA) receptor, which is important for certain forms of neural plasticity in hippocampus. Indeed, the integrity of these components of hippocampal neuronal machinery is consistent with the stereological evidence that neuron numbers are preserved in this system during normal ageing.

The findings in this line of research indicate substantial structural integrity in a system where, until recently, cell loss was considered to be a major contributing factor to functional decline during the normal ageing process. The absence of neuron loss in this structure in aged subjects, contrasts with an attrition confirmed in brains from patients with Alzheimer's disease (West et al. 1994). Nonetheless, the behavioural decline in hippocampal-dependent learning demonstrated in the animal model would seem to indicate that other effects of ageing compromise the function of the medial temporal lobe system. Indeed, a number of alterations that could provide a basis for the emergence of behavioural deficits are evident within this circuitry. For example, stereological analyses have revealed a loss of synaptic contacts at certain sites within the hippocampal formation of aged rats (Geinesman et al. 1992). Impaired long-term plasticity is also evident in the aged rat hippocampus (Barnes 1979). In addition, although total numbers of principal neurons within the hippocampal formation are preserved, neurodegeneration of subcortical neurons, i.e. basal forebrain cholinergic cells, that provide input to the hippocampus has also been confirmed in aged rats (Fischer et al. 1991). Such effects of ageing may provide a basis for diminished proficiency in functions dependent on the hippocampal system. Indeed, a number of alterations in the hippocampal system during ageing have been found to correlate with the severity of impairment in hippocampal-dependent learning and memory, such as that observed in the spatial learning model (for review Gallagher et al. 1995).

\section{RELATIONSHIP OF GHOLINERGIC NEURON LOSS TO GOGNITIVE IMPAIR MENT}

To establish the functional significance of a specific alteration observed in the brain during ageing, two general approaches are taken. By one approach, experimental induction of the alteration observed in ageing would be expected to produce deficits like those encountered in aged subjects. As a complementary approach, therapeutic treatments designed to remedy or compensate for a neurobiological alteration would be expected to improve deficits caused by that 

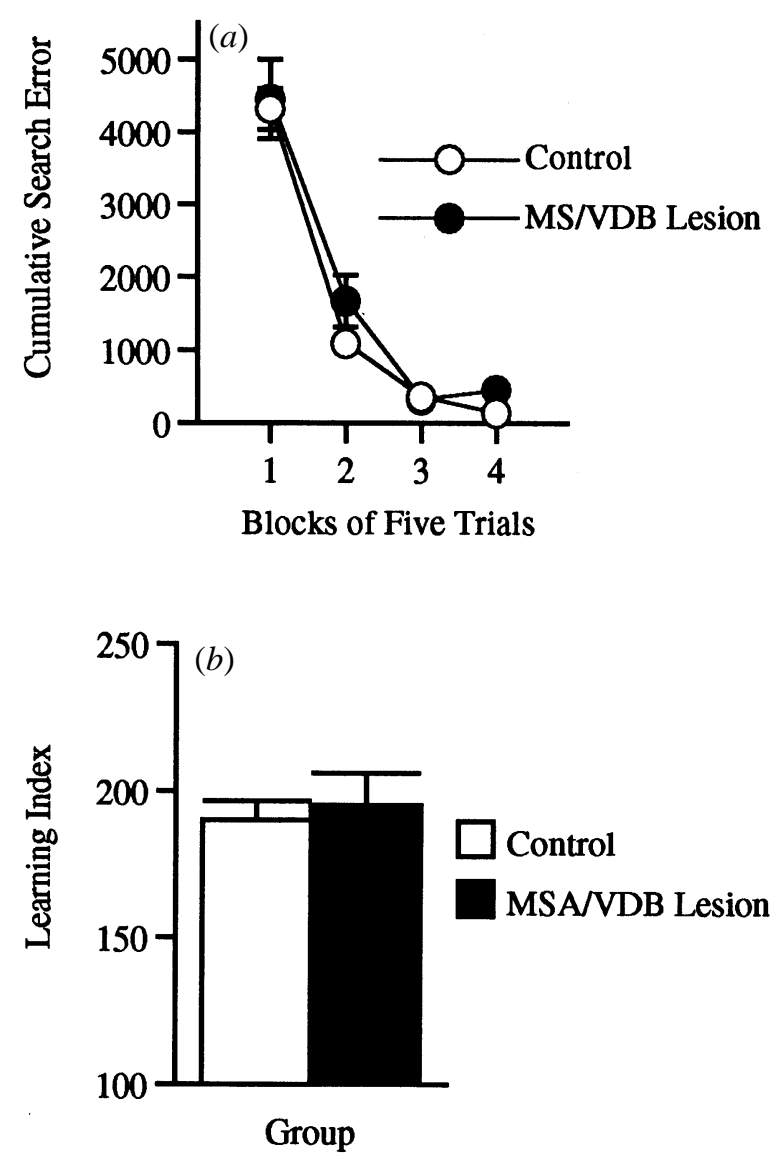

Figure 2. Effects of immunotoxic lesions of the cholinergic neurons that project to hippocampus on spatial learning in the water maze (Baxter et al. 1995). Both control and lesioned rats show comparable learning during training trials $(a)$ and probe trials $(b)$.

alteration. We now turn to some unexpected findings from studies designed to examine the contribution of cholinergic deficiency to the decline in hippocampaldependent spatial learning observed in aged rats.

A deficiency in the cholinergic system has been of long-standing interest as a basis for memory impairment in ageing and age-related pathological conditions, such as Alzheimer's disease. The basal forebrain cholinergic system consists of a grouping of large neurons that extends from the medial septum through the diagonal band of Broca to the nucleus basalis of Meynert, also including the sublenticular substantia innominata, particularly in rodent brain (Wainer \& Mesulam 1990). Neurons situated more rostrally, in the medial septum and vertical limb of the diagonal band (MS/vDB), predominantly innervate the hippocampal formation. At more caudal levels, cholinergic neurons innervate neocortex and the amygdala complex. These cholinergic neurons undergo substantial degeneration in Alzheimer's disease (Coyle et al. 1983; Davies \& Maloney 1976) and to a lesser extent in normal ageing (Bartus et al. 1985). In the rodent model of hippocampal-dependent function, a number of laboratories have reported that deterioration of these cholinergic neurons is reliably correlated with age-related decline in spatial learning in rats (for reviews, see Fisher et al. 1991; Gallagher et al. 1995). Such findings are consistent with correlations also reported between the degree of cholinergic loss and severity of cognitive decline in dementia (Bartus et al. 1985). All of these observations have been viewed as lending support to the concept that memory impairment in ageing and dementia is attributable to a cholinergic deficiency. By this view, it would be expected that removal of cholinergic neurons would be sufficient to cause deficits that resemble those encountered in ageing and, to a more severe degree, in dementia. A test of this prediction has become possible by the recent development of a selective toxin for cholinergic neurons.

The immunotoxin, 192 IgG-saporin, is composed of a monoclonal antibody to the 'low-affinity' p75 NGF receptor $\left({ }_{\mathrm{L}} \mathrm{NGFr}\right)$ coupled to a ribosome-inactivating cytotoxin, saporin (Wiley et al. 1991). In the basal forebrain of rats, the ${ }_{\mathrm{L}} \mathrm{NGFr}$ is selectively expressed on cholinergic neurons, so direct infusion of 192 IgGsaporin into basal forebrain nuclei produces selective lesioning of cholinergic neurons, sparing non-cholinergic neurons at the lesion site. The lesion methods using microinjection of $192 \mathrm{IgG}$-saporin into the MS/ vDB in the studies described here produced $>90 \%$ reduction of ChAT activity in the hippocampal formation. If deficits in spatial learning in aged animals are the result (entirely or partially) of loss of cholinergic input to the hippocampus, young animals with this immunotoxic lesion should have impairments in spatial learning that are similar to those seen in aged rats. This hypothesis was examined by testing young immunolesioned rats in the same protocol that has been well-characterized in our laboratory for examining the deficits in spatial learning that occur in ageing (Baxter et al. 1995). The results from that experiment are shown in figure 2.

Surprisingly, rats with removal of the septo-hippocampal cholinergic projection acquire the spatial learning task as readily as intact rats. The failure to find substantial impairment in the spatial learning water maze task, as well as in other assessments of learning and memory, has also been reported by other investigators using 192 IgG-saporin to produce selective cholinergic lesions of components of the basal forebrain system that innervate hippocampus (Berger-Sweeney et al. 1994; Torres et al. 1994).

A selective loss of cholinergic neurons that innervate the hippocampal formation might not provide an adequate model for the effects of ageing on the basal forebrain cholinergic system. If the entire basal forebrain cholinergic system, with its collective projections to hippocampus, amygdala and cortex normally provides a coordinated regulation of certain processing capacities in limbic/cortical circuitry, then more complete removal of the entire basal forebrain cholinergic system, rather than components of it, may provide a better basis for revealing its function. More extensive lesions of the basal forebrain cholinergic system may also better mimic the pattern of loss that occurs during ageing and age-related pathological conditions. Deficiencies in the components of this system that innervate cortex are typically seen along with loss of the septo-hippocampal cholinergic projection. In a more recent study, microinjections were made throughout the rostral/caudal extent of the 


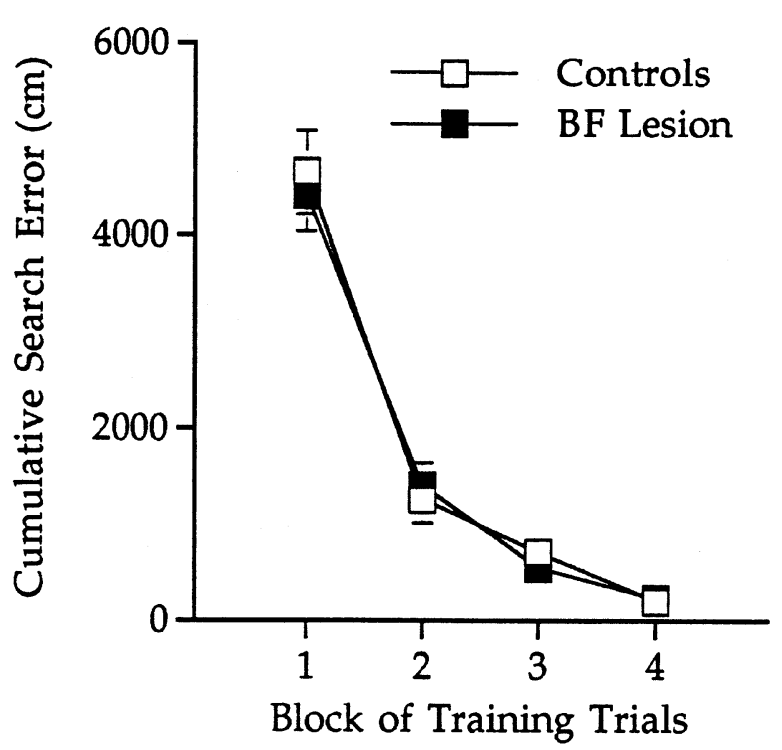

Figure 3. Effects of combined lesions of the MS/VDB and $\mathrm{nBM} / \mathrm{SI}$ (basal forebrain (BF) lesion) on spatial learning in the water maze (Baxter et al. 1996). Both control and lesioned rats show comparable learning.

basal forebrain system. Despite this extensive removal of the basal forebrain cholinergic system, the data in figure 3 show that spatial learning was acquired normally by the immunolesioned rats (Baxter et al. 1996).

Before concluding that loss of the integrity of cholinergic input to hippocampus is not sufficient to cause spatial learning impairment, we thought it important to examine the effects of removing septo-hippocampal cholinergic neurons in aged rats. Against the background of other neurobiological changes in the hippocampus of aged rats, removal of these neurons might produce a deficit where none is seen in young animals. For this purpose, young and aged rats were initially screened in our standard spatial learning protocol to select matched groups for the control and lesion conditions. Aged rats with relatively preserved learning ability were chosen so as to avoid a floor effect in our assessment of the lesion, but we also included some aged rats with marginal spatial learning impairment to determine if these rats might be more vulnerable to the effects of cholinergic lesions. Nonetheless, we found no difference in this study between lesion and control groups, irrespective of age or preoperative performance (see Baxter \& Gallagher 1996, figure 4).

The experiments described thus far indicate that removal of basal forebrain cholinergic neurons is not sufficient to produce spatial learning impairment. It might be argued, however, that the contribution of these neurons to behaviour might only be detected if additional effects that coincide with greater cognitive impairment in ageing were also reproduced. Thus, the behavioural capacity of aged rats with relatively intact or only mild impairment would not be further compromised by a selective cholinergic lesion. Other studies, however, argue against this view that a cholinergic lesion, by itself, is not sufficient to cause behavioural impairment. Indeed, young rats with selective lesions
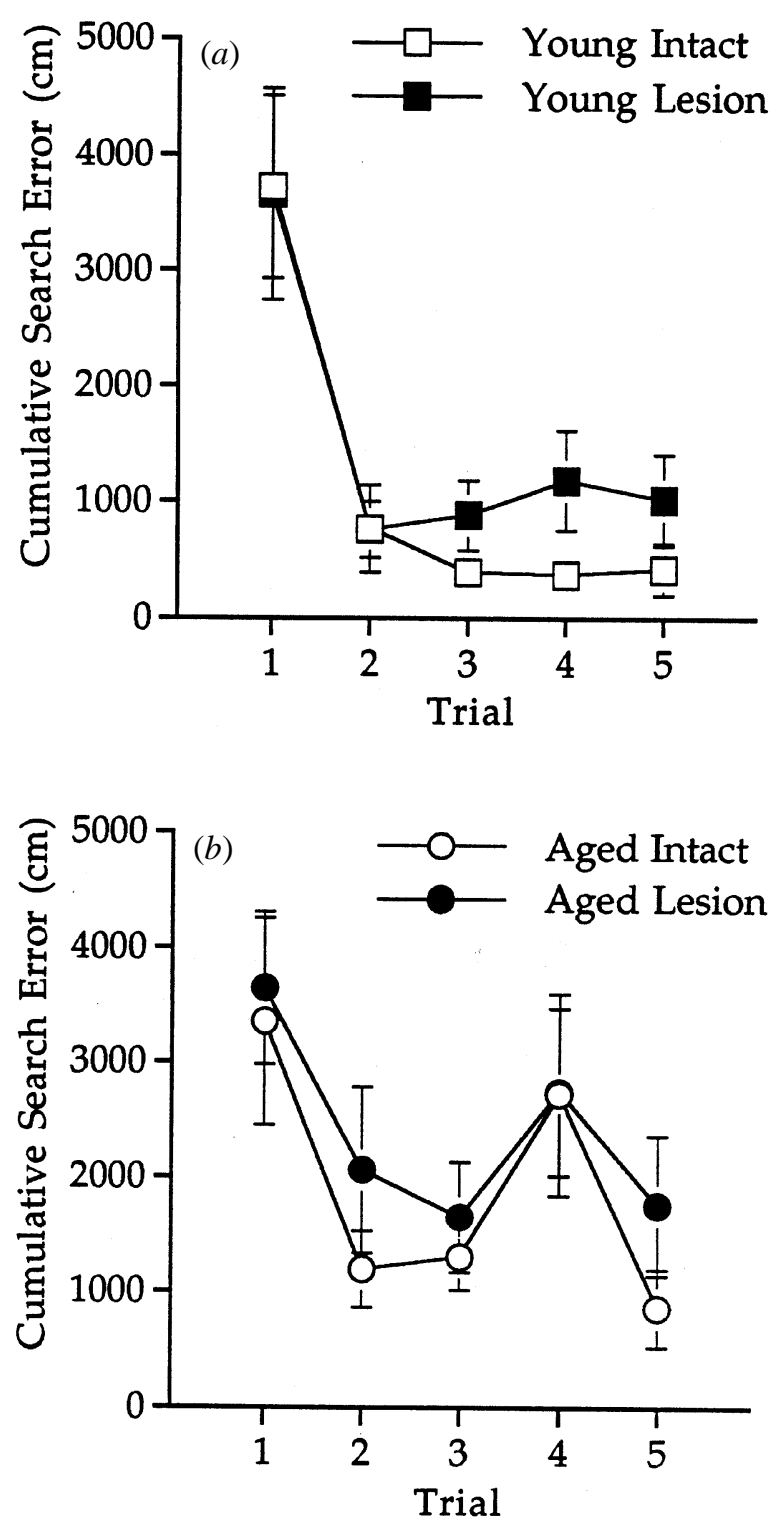

Figure 4. Effects of immunolesions removing cholinergic input to the hippocampus on spatial learning in young $(a)$ and aged rats $(b)$. All rats were tested in a standard water maze protocol (Gallagher et al. 1993) prior to assignment to lesion or control groups; rats within an age group were matched for preoperative spatial learning ability and aged rats with severely impaired spatial learning were excluded. Following surgery, spatial learning was tested in a new maze with different extra-maze cues. There was no significant effect of the immunolesion on spatial learning in either young or aged rats (Baxter \& Gallagher 1996).

of the basal forebrain cholinergic system are impaired on assessments in other behavioural domains. In particular, selective removal of these cholinergic neurons in young rats using the toxin $192 \mathrm{IgG}$-saporin is sufficient to cause substantial deficits in tasks that primarily assess the regulation of attentional processes (Baxter $e t$ al. 1997; Chiba et al. 1995, 1997; and see Baxter \& Gallagher 1997, for a review). Moreover, those findings indicate that lesions of different components of this system (innervating hippocampus and cortex) impair attentional processes in distinctive ways. These new findings add support to a shifting focus on the contribution of 
cholinergic deficiency in ageing and Alzheimer's disease to impairments in attentional processes (Jones et al. 1995; Moore et al. 1992; Mouloua \& Parasuraman 1995; Parasuraman et al. 1992; Parasuraman \& Haxby 1993).

If removal of cholinergic neurons in the $\mathrm{MS} / \mathrm{vDB}$ is ineffective for producing spatial learning deficits in young and aged rats, how are correlations between cholinergic degeneration in the MS/vDB and agerelated spatial learning impairments to be interpreted? Such correlations have suggested that cholinergic deficiency contributes to the functional loss assessed in the animal model of spatial learning. An alternative view of such correlations is that age-related changes in cholinergic neurons reflect a separate, underlying condition in the hippocampus that itself causes spatial learning impairment. Indeed, selective neurotoxic lesions of hippocampus (unlike removal of the septohippocampal cholinergic neurons) do reproduce age-related deficits in spatial learning (Gallagher \& Holland 1992). Consistent with this possibility, there is also extensive evidence for age-related changes in the hippocampus other than those alterations found in the cholinergic projection system (Barnes 1979; Geinesman et al. 1992; and Gallagher \& Rapp 1997, for a review). By this view, the condition of the aged hippocampus that underlies spatial learning impairment (or some subset of those changes) would be associated only secondarily with deterioration in the cholinergic system.

\section{GONGLUSION}

Animal models have provided a rich source of information about the neurobiological basis of memory. This presentation has focused on new insights from the use of an animal model of spatial learning and memory to study the neurobiological basis of cognitive decline in ageing. Recent research has shown that identified impairment in learning and memory in aged rats is not tied to frank neurodegeneration in the hippocampal system. As an extension of the study of spatial learning in aged rodents, research using this model to assess experimental treatments that reproduce specific features of neurobiological ageing in young animals, such as deterioration in the basal forebrain cholinergic projection to hippocampus, have led to a reconceptualization of the functional significance of age-related deterioration in this system. A clear implication from this research is that interventions designed to address cholinergic deficiency in ageing and Alzheimer's disease might be more therapeutic in treating deficits in attention than deficits in memory (see Sahakian et al. (1993) for research consistent with this proposal). Further use of well-characterized animal models will undoubtedly play a central role in determining those neurobiological effects of ageing that are important substrates for specific aspects of cognitive decline in ageing and in pathological conditions that occur in the elderly population. Such research is of clear fundamental importance in the development of appropriate and effective treatments in those conditions.

Supported by grants from the National Institute of Mental Health (KO5-MH01149) and National Institute on Ageing (POl-AG09973).

\section{REFERENGES}

Barnes, C. A. 1979 Memory deficits associated with senescence: neurophysiological and behavioral study in the rat. 7. Comp. Physiol. Psych. 93, 7-104.

Bartus, R. T., Dean, R. L., Pontecorvo, M. J. \& Flicker, C. 1985 The cholinergic hypothesis: a historical overview, current perspective, and future directions. Ann. NY Acad. Sci. 444, 332-358.

Baxter, M. G. \& Gallagher, M. 1996 Intact spatial learning in both young and aged rats following selective removal of hippocampal cholinergic input. Behav. Neurosci. 110, 460-467.

Baxter, M. G. \& Gallagher, M. 1997 Cognitive effects of selective loss of basal forebrain cholinergic neurons: implications for cholinergic therapies of Alzheimer's disease. In Pharmacological treatment of Alzheimer's disease: molecular and neurobiological foundations (ed. J. D. Brioni \& M. W. Decker). pp. 87-103. New York: Wiley.

Baxter, M. G., Gallagher, M. \& Holland, P. C. 1997 Disruption of decremental attentional processing by selective removal of hippocampal cholinergic input. F. Neurosci. 17, 5230-5236.

Baxter, M. G., Bucci, D. J., Gorman, L. K., Wiley, R.G. \& Gallagher, M. 1995 Selective immunotoxic lesions of basal forebrain cholinergic cells: effects on learning and memory in rats. Behav. Neurosci. 109, 714-722.

Baxter, M. G., Bucci, D. J., Sobel, T. J., Williams, M. J., Gorman, L. K. \& Gallagher, M. 1996 Intact spatial learning following lesions of basal forebrain cholinergic neurons. Neuro Report 7, 1417-1420.

Berger-Sweeney, J., Heckers, S., Mesulam, M.-M., Wiley, R. G., Lappi, D. A. \& Sharma, M. 1994 Differential effects on spatial navigation of immunotoxin-induced cholinergic lesions of the medial septal area and nucleus basalis magnocellularis. F. Neurosci. 14, 4507-4519.

Chiba, A. A., Bucci, D. J., Holland, P. C. \& Gallagher, M. 1995 Basal forebrain cholinergic lesions disrupt increments but not decrements in conditioned stimulus processing. $\mathcal{F}$. Neurosci. 15, 7315-7322.

Chiba, A. A., Bushnell, P. J., Oshiro, W. M. \& Gallagher, M. 1997 Altered selective attention in rats with cholinergic lesions of the substantia innominata. NeuroReport. (In the press.)

Chouinard, M. L., Gallagher, M., Uasuda, R. P., Wolfe, B. B. \& McKinney, M. 1995 Hippocampal muscarinic receptor function in spatial learning impaired aged rats. Neurobiol. Ageing 16, 955-963.

Coyle, J. T., Price, D. L. \& DeLong, M. R. 1983 Alzheimer's disease: a disorder of cortical cholinergic innvervation. Science 219, 1184-1190.

Davies, P. \& Maloney, A. J. F. 1976 Selective loss of central cholinergic neurons in Alzheimer's disease. Lancet 2, 1403.

Fischer, W., Chen, K. S., Gage, F. H. \& Björklund, A. 1991 Progressive decline in spatial learning and integrity of forebrain cholinergic neurons in rats during ageing. Neurobiol. Ageing 13, 9-23.

Gage, F. H., Dunnett, S. B. \& Björklund, A. 1984 Spatial learning and motor deficits in aged rats. Neurobiol. Ageing $\mathbf{5}$, 43-48.

Gallagher, M. \& Holland, P. C. 1992 Preserved configural learning and spatial learning impairment in rats with hippocampal damage. Hippocampus 2, 81-88.

Gallagher, M. \& Rapp, P. R. 1997 The use of animal models to study the effects of aging on cognition. A. Rev. Psychol. 48, 339-370.

Gallagher, M., Burwell, R. \& Burchinal, M. 1993 Severity of spatial learning impairment in ageing: development of a learning index for performance in the Morris water maze. Behav. Neurosci. 107, 618-626.

Geinesman, Y., deToledo-Morrell, L., Morrell, F., Persina, I. S. \& Rossi, M. 1992 Age-related loss of axcospinous synapses formed by two afferent systems in the rat dentate 
gyrus as revealed by the unbiased stereological dissector technique. Hippocampus 2, 437-444.

Hyman, B. T., Van Hoesen, G. W. \& Damasio, A. R. 1987 Alzheimer's disease: glutamate depletion in the hippocampal perforant pathway zone. Ann. Neurol. 22, 37-40.

Hyman, B. T., Van Hoesen, G. W., Damasio, A. R. \& Barnes, G. L. 1984 Alzheimer's disease: cell specific pathology isolates the hippocampal formation. Science 225, 1168-1170.

Issa, A. M., Rowe, W., Gauthier, S. \& Meaney, M. J. 1990 Hypothalamic-pituitary-adrenal activity in aged, cognitively impaired and cognitively unimpaired rats. $\mathcal{f}$ Neurosci. 10, 3247-3254.

Jones, D. N. C., Barnes, J. C., Kirkby, D. L. \& Higgins, G. A. 1995 Age-associated impairments in a test of attention: evidence for involvement of cholinergic systems. F. Neurosci. 15, 7282-7292.

Landfield, P. W., Baskin, R. K. \& Pitler, T. A. 1981 Brain ageing correlates: retardation by hormonal-pharmacological treatments. Science 214, 581-584.

Meaney, M. J., Aitken, D. H., van Berkel, C., Bhatnagar, S. \& Sapolsky, R. M. 1988 Effect of neonatal handling on agerelated impairments associated with the hippocampus. Science 239, 766-768.

Moore, H., Dudchenko, P., Bruno, J. P. \& Sarter, M. 1992 Toward modeling age-related changes of attentional abilities in rats: simple and choice reaction times tasks and vigilance. Neurobiol. Ageing 13, 759-772.

Morris, R. G. M., Garrud, P., Rawlins, J. N. P. \& O'Keefe, J. 1982 Place navigation impaired in rats with hippocampal lesions. Nature 297, 681-683.

Mouloua, M. \& Parasuraman, R. 1995 Ageing and cognitive vigilance: effects of spatial uncertainty and event rate. Expl Ageing Res. 21, 7-32.

Parasuraman, R., Greenwood, P. M., Haxby, J. V. \& Grady C. L. 1992 Visuospatial attention in dementia of the Alzheimer type. Brain 115, 711-733.

Parasuraman, R. \& Haxby, J. V. 1993 Attention and brain function in Alzheimer's disease: a review. Neuropsychologia 7, 242-272.
Rapp, P. R. \& Amaral, D. G. 1992 Individual differences in the cognitive and neurobiological consequences of normal ageing. Trends Neurosci. 15, 340-345.

Rapp, P. R. \& Gallagher, M. 1996 Preserved neuron number in the hippocampus of aged rats with spatial learning deficits. Proc. Natn. Acad. Sci. USA 93, 9926-9930.

Rasmussen, T., Schielmann, T., Sorenson, J. C., Zimmer, J. \& West, M. J. 1996 Memory impaired aged rats: no loss of principal hippocampal and subicular neurons. Neurobiol. Ageing 17, 143-147.

Sahakian, B. J., Owen, A. M., Morant, N. J., Eagger, S. A., Boddington, S., Crayton, L., Crockford, H. A., Crooks, M., Hill, K. \& Levy, R. 1993 Further analysis of the cognitive effects of tetrahydroaminoacridine (THA) in Alzheimer's disease: assessment of attentional and mnemonic function using CANTAB. Psychopharmacology 110, 395-401.

Torres, E. M., Perry, T. A., Blokland, A., Wilkinson, L. S., Wiley, R. G., Lappi, D. A. \& Dunnett, S. B. 1994 Behavioural, histochemical and biochemical consequences of selective immunolesions in discrete regions of the basal forebrain cholinergic system. Neuroscience 63, 95-122.

Wainer, B. H. \& Mesulam, M.-M. 1990 Ascending cholinergic pathways in the rat brain. In Brain cholinergic systems (ed. M. Steriade \& D. Biesold), pp. 65-119. Oxford University Press.

West, M. 1993 Regionally specific loss of neurons in the ageing hippocampus. Neurobiol. Ageing 14, 287-293.

West, M., Amaral, D. G. \& Rapp, P. R. 1993 Preserved hippocampal cell number in aged monkeys with recognition memory deficits. Soc. Neurosci. Abstr. 19, 599.

West, M., Goleman, P. D., Flood, D. G. \& Troncoso, J. C. 1994 Differences in the pattern of hippocampal neuronal loss in normal ageing and Alzheimer's disease. Lancet 344, 769-772.

Wiley, R. G., Oeltmann, T. N. \& Lappi, D. A. 1991 Immunolesioning: selective destruction of neurons using immunotoxin to rat NGF receptor. Brain Res. 562, 149-153. 
